\title{
A Study of Serum Hs CRP Levels in Osteoarthritis Knee with Clinical and Radiological Correlation
}

\author{
Dr. S.Sivakumar ${ }^{1}$ M.D., Dr. M.A.Shakeelahmed ${ }^{2}$ M.D., \\ Dr. S.Senthilkumar ${ }^{3}$ M.D., \\ ${ }^{1}$ Associate Professor, Department Of General Medicine, Government Thiruvannamalai \\ Medical College And Hospital \\ ${ }^{2}$ Associate Professor, Department Of General Medicine, Government Thiruvannamalai \\ Medical College And Hospital \\ ${ }^{3}$ Senior Resident, Department Of General Medicine, Government Thiruvannamalai \\ Medical College And Hospital
}

\begin{abstract}
Introduction: Osteoarthritis is the most common rheumatologic disease of old age. It is a slowly progressive degenerative disease leading to major restriction of activities of daily living in the elderly. Symptoms of Osteoarthritis include pain, stiffness, restriction of joint movement and deformity of joint.

Osteoarthritis usually affects knee, hip, hands, Spine rarely ankle. Radiographic evidence of OA outnumbers the clinically evident Osteoarthritis.

There is no definite cure for OA. Wide variety of treatment measures include weight reduction, modification of activities to reduce stress and load on the joint, Pharmacotherapy such as analgesics, judicious, use of NSAIDS or intraarticular steroid injection.

Total joint replacement is the treatment of choice but could not be done in all patients.

Although osteoarthritis is a non inflammatory disease, some inflammatory process occurs in the joint causing pain.

Recent investigation focus on various biomarkers to assess osteoarthritis severity and to classify disease progression.

The aim of our study is to consider one such marker of inflammation hsCRP as a tool of disease severity, x-ray grading of knee joint is studied in comparison with hsCRP levels and patient symptoms.

Our study design is cross sectional study designed to assess the correlation between severities with its symptoms, $X$-ray findings and inflammatory markers. i.e. hsCRP

Aims and Objectives:

- To study serum HS CRP levels in sample of osteoarthritis patients.

- To assess Hs CRP level in correlation with clinical symptoms and radiological signs.

Materials and methods: Patients fount to have clinical evidence of osteoarthritis of knee are selected and subjected to cross sectional study. They are subjected to detailed history, clinical examination, and radiographic evaluation of knee along with high sensitive C-reactive protein. Then it is planned to assess the correlation if any exist between clinical symptoms, radiological findings and hsCRP levels.
\end{abstract}

Study centre: Government Thiruvannamalai Medical College and Hospital, Thiruvannamalai

Duration of the study: 6 Months

Study design: Observational study

Sample size: 60 patients

Data collection and methods: Patients have their history taken according to a questionnaire and subjected to clinical examination and investigations.

Product/procedure/ investigation details: $X$-ray knee, hsCRP levels.

Inclusion criteria: Idiopathic osteoarthritis, Patient with evidence of clinical osteoarthritis, Patient aged $40 \mathrm{Yrs}$ and above.

Exclusion criteria: Patients with inflammatory rheumatologic disorders like RA, SLE, Crystal arthropathies, Reactive arthritis, Cardiovascular disease, COPD, Ulcerative colitis, Chronic liver disease, Chronic kidney disease, Previous history of local intraarticular steroid injection.

Statistical methods: The statistical analysis is done

Conclusion:

In our study of hsCRP levels in osteoarthritis patients, the conclusions are

- Osteoarthritis is more prevalent in females.

- $\quad$ hSCRP levels are modestly elevated in many patients particularly those with local symptoms .

- $\quad$ hSCRP levels rise with increasing pain scores.

DOI: 10.9790/0853-1509070109 www.iosrjournals.org 
- $\quad$ hSCRP levels do not correlate with $x$-ray grading .

- hsCRP levels have minimal correlation with body mass index.

- Patients with high levels of hsCRP are likely to progress to advance disease.

Keywords: Osteoarthritis, hsCRP

\section{Introduction}

Osteoarthritis is the most common rheumatologic disease of old age. It is a slowly progressive degenerative disease leading to major restriction of activities of daily living in the elderly. Symptoms of osteoarthritis include pain, stiffness, restriction of joint movement and deformity of joint.

Osteoarthritis usually affects knee, hip, hands, Spine rarely ankle. Radiographic evidence of OA outnumbers the clinically evident osteoarthritis.

There is no definite cure for OA. Wide variety treatment measures include weight reduction, modification of activities to reduce stress and load on the joint, Pharmacotherapy such as analgesics, judicious, use of NSAIDS or intraarticular steroid injection. Total joint replacement is the treatment of choice but could not be done in all patients.

Although osteoarthritis is a non inflammatory disease, some inflammatory process occurs in the joint causing pain.

Recent investigation focus on various biomarkers to assess osteoarthritis severity and to classify disease progression.

The aim of our study is to consider one such marker of inflammation hsCRP as a tool of disease severity, $\mathrm{x}$-ray grading of knee joint is studied in comparison with hsCRP levels and patient symptoms.

While treating osteoarthritis patient the treating physician should

- Create an awareness of the disease and educate the patient to change the activities of daily living for secondary prevention of complication.

- To inform the patient about various treatment options available. Individual risk and benefits of each modality.

- To attain optimal quality of life with appropriate available measures

- To provide adequate rehabilitation measures for selected patients.

Our study design is cross sectional study designed to assess the correlation between severities with its symptoms. X-ray treatment and inflammatory marks. i.e. hsCRP

\section{Aims and objectives}

- To study serum hsCRP levels in sample of Osteoarthritis patient.

- To assess hsCRP level in correlation with clinical symptoms and radiological signs.

\section{Biomarkers of $\mathrm{OA}$}

COMP, Cartilage Intermediate Layer protein (CILP), Cartilage Link Protein Matrilin and Minor Collagen and Hyaluronic Acid are synthesized and degraded in OA in excess levels.

- In healthy cartilage this molecules have relatively slow turnover - These markers of cartilage matrix synthesis and degradation can be used as biomarkers of OA

- $\quad$ Biomarkers of OA can be used to find

- Burden of disease

- Investigative markers

- Prognostic markers

- Efficacy and effectiveness of intervention

- Diagnostic

Examples of burden of disease and prognosis includes serum COMP, Serum HA and Urinary CTX II

- One study of patients with OA knee compared MRI findings with level of serum HA, Osteocalcin, Cartilage Glycoprotein 39, COMP and Urine c-Terminal telopeptide of type 2 collagen. Findings suggested one time measurement of HA or short term increase in CTX II would identify patient at greater risk for OA progression ${ }^{7}$.

\section{$\mathbf{C R P} *(\mathbf{C}$ - reactive protein $)$}

- OA is not considered as inflammatory disease but it definitely involves inflammatory process and it provides promise for biomarkers

- Elevated levels of CRP appear to be predictive of radiographic progression of long term knee OA. 
- In a study of 1025 women, higher CRP levels are associated with statistically significant increase in both prevalent and incident $\mathrm{OA}$ and greater knee severity

- Bilateral OA knee patient has comparatively higher CRP levels than unilateral OA

- Body mass index should be taken into account for CRP levels ${ }^{8}$.

- CRP are modestly but significantly elevated in OA early knee OA and

Predictive of disease progression

\section{COMP (Cartilage Oligomeric Matrix Protein)}

- COMP is a non-collagenous ECM protein synthesized by cartilage and synovium is abundant in articular cartilage $^{9,10,11,12}$

- COMP levels helpful in assessing presence and progression of OA

- Synovial fluid COMP levels elevated in patients with knee ligaments injury or meniscal injury.

- Serum COMP levels are higher in patients with rapidly progressing joint damage

\section{Hyaluronic Acid}

- HA is a cartilage degradation marker and can be detected in serum and synovial fluid ${ }^{13}$.

- HA levels reflect activity of synovium but proteoglycan levels reflect turnover of cartilage.

- Higher serum HA levels have been correlated with number of joints involved and degree of clinical severity.

- Serum HA levels also serve as predictor of OA disease progression.

\section{Imaging:}

- Conventional radiography is inexpensive and widely available, used to confirm the diagnosis and exclude other conditions.

- X-ray in OA show osteophytes, joint space narrowing, osteosclerosis and subchondral bone cysts.

- Kellgren-lawrence grading system commonly used for research purpose range from 0 (no osteophytes; no joint space narrowing) to 4 (severe joint space narrowing with subchondral sclerosis).

- K-L grade 2 is usually considered diagnosis of OA.

- K-L grading is much less helpful in OA of hip.

- Osteoarthritis Research Society International grading system (OARSI) view osteophytes and joint space narrowing separately and assign separate scores ${ }^{15}$.

- Imaging of knees should involve both knee joint and in weight bearing state.

- Lateral, poster anterior or sunrise views may be necessary.

\section{Kellgren And Lawrence System Classification Of Oa Knee}

KL system is a method is a classifying the severity of knee OA using five grades:

- Grade 0: no radiographic features of OA present

- Grade 1: doubtful joint space narrowing (JSN) and possible osteophytic lipping.

- Grade 2: definite osteophytes and possible JSN on antero-posterior weight bearing radiograph

- Grade 3: multiple osteophytes, definite JSN, Sclerosis, possible bony deformity

- Grade 4: large osteophytes, marked JSN, severe sclerosis and definitely bony deformity

KL Grade 2

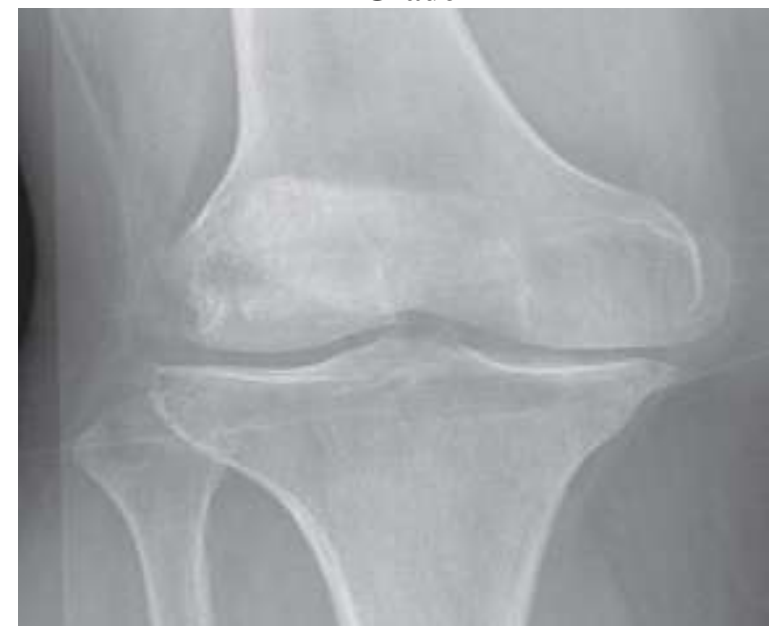


KL Grade 3

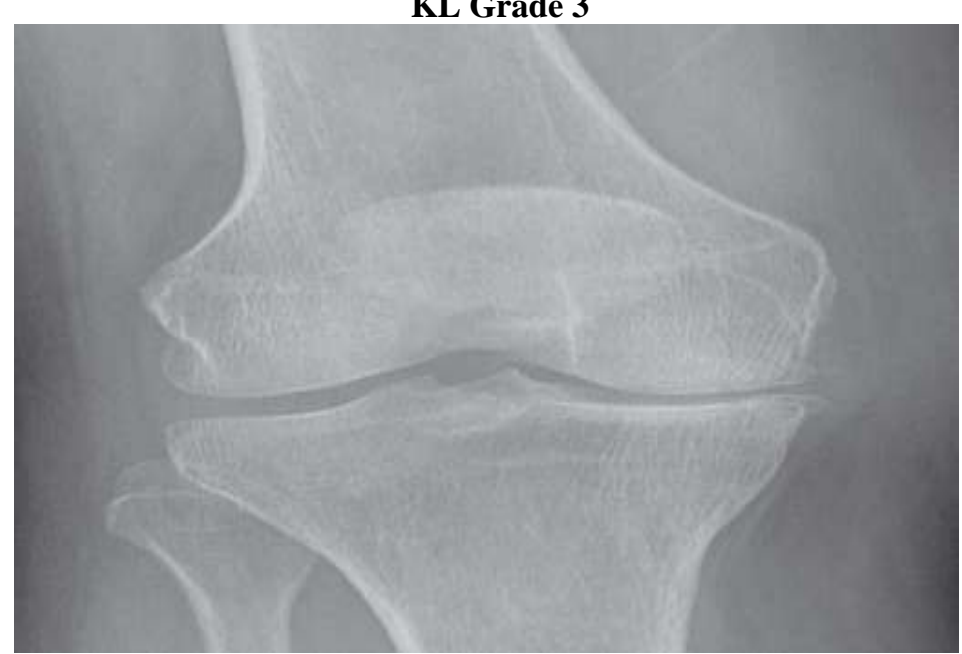

KL Grade 4
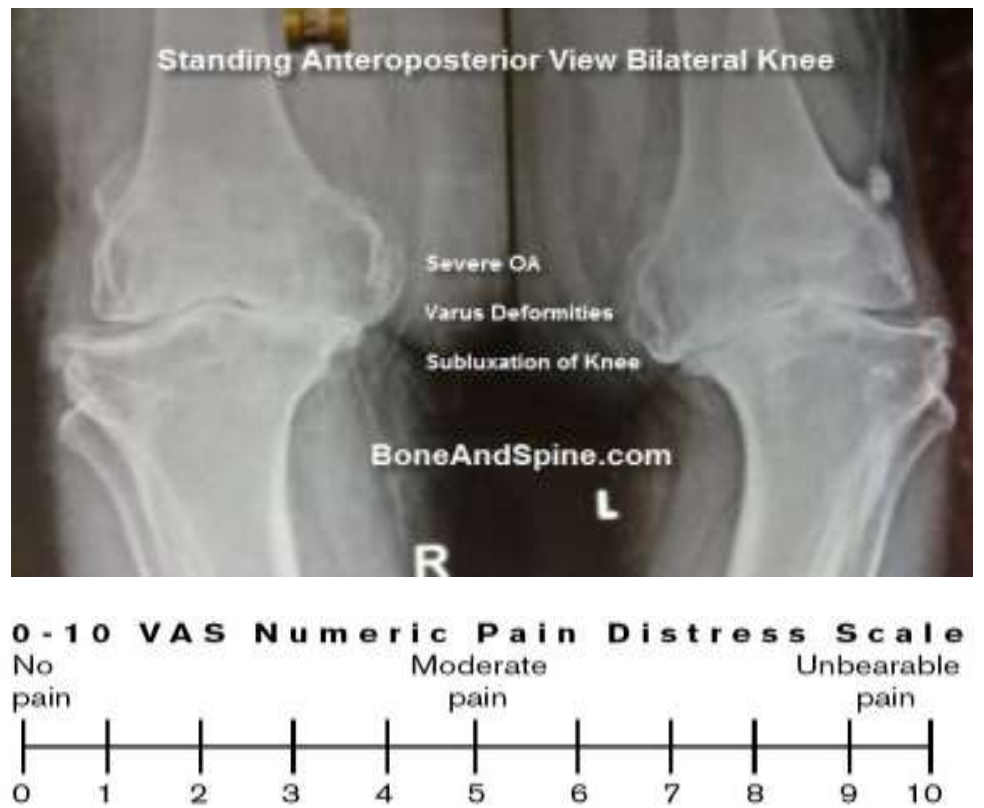

Other causes of hsCRP elevation

- Recurrent coronary events

- COPD

- Rheumatoid arthritis

- Asthma

- SLE

- Diabetes mellitus

- Smoking

\section{Selection of volunteers:}

IV. Materials and methods

On patient found to have clinical evidence of osteoarthritis of knee are selected and subjected to cross sectional study. They are subjected to detailed history, clinical examination, and radiographic evaluation of knee along with high sensitive C-reactive protein. Then it is planned to assess the correlation if any exist between clinical symptoms, radiological findings and hsCRP levels.

Study centre: Government Thiruvannamalai Medical College and Hospital, Thiruvannamalai

Duration of the study: 6 Months

Study design: Observational study

Sample size: 60 patients 
Data collection and methods: Patients have their history taken according to a questionnaire and subjected to clinical examination and investigations.

Product/Procedure/Investigation details: X-ray knee, hsCRP levels.

\section{Inclusion criteria}

- Idiopathic osteoarthritis

- Patient with evidence of clinical osteoarthritis

- Patient aged 40 yrs and above

\section{Exclusion criteria}

- Patients with inflammatory rheumatologic disorders RA, SLE, Crystal arthropathies, Reactive arthritis

- Cardiovascular disease, COPD, Ulcerative colitis.

- Chronic Liver Disease

- Chronic Kidney Diseases

- Previous history of local intraarticular steroid injection

The statistical analysis is done

\section{Statistical Methods}

\section{Discussion}

In our study 60 patients found to have osteoarthritis of knee were enrolled as participants. Osteoarthritis patients are diagnosed by history and clinical examination. Our study was conducted between February 2016 and July 2016. Aims of the study and study design were explained and institutional ethics clearance obtained.

Osteoarthritis patient enrolled and the purpose of study explained to the participants. After the informed consent of the participants detailed history and clinical examination were done. Patients who were satisfying ACR criteria for osteoarthritis of knee were considered for study. Patients with evidence of inflammatory arthritis were excluded, so also there with Diabetes mellitus, Hypertension, Chronic liver disease, Chronic kidney disease were excluded from the study because these factors may interfere with our study outcome.

Symptoms of the patients such as pain, stiffness, swelling and disability were enquired. Pain was graded by visual analog scale (VAS) graded from 0 to 10 . (No pain on the left side of scale to extreme pain on right).

All the patients were examined clinically and their body mass index calculated, examined for effusion and tenderness and joint deformity.

$\mathrm{X}$-Ray of both knee joints taken in standing posture, grading of X-Ray changes done by Kellgren and Lawrence system which is from 0 to 4 . All our patients were more than K-L grade 2.

Blood sample $5 \mathrm{ml}$ collected from each patient and centrifuged to collect the plasma and stored in frozen state. After collecting all samples hsCRP levels estimated by ELISA technique after making adequate dilution.

\section{Age Distribution Chart}

\section{AGE DISTRIBUTION CHART}

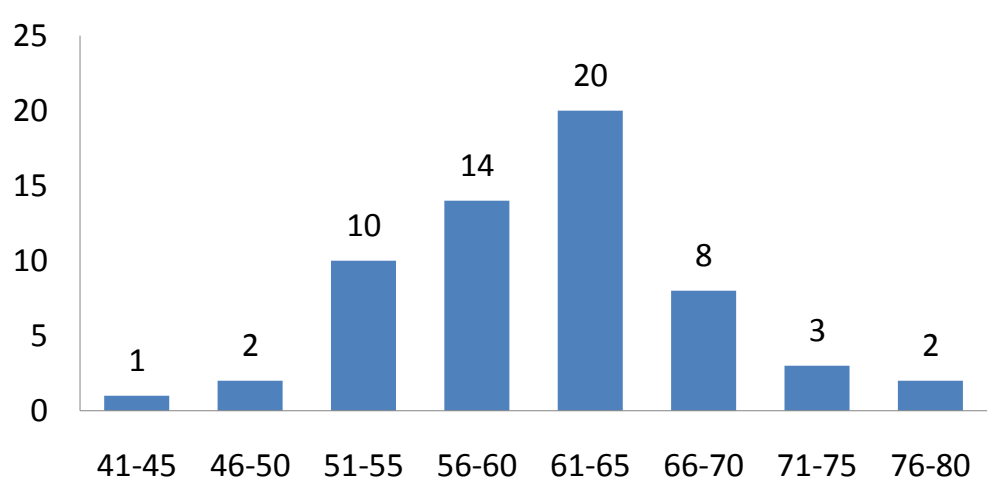




\section{Untitled 2}

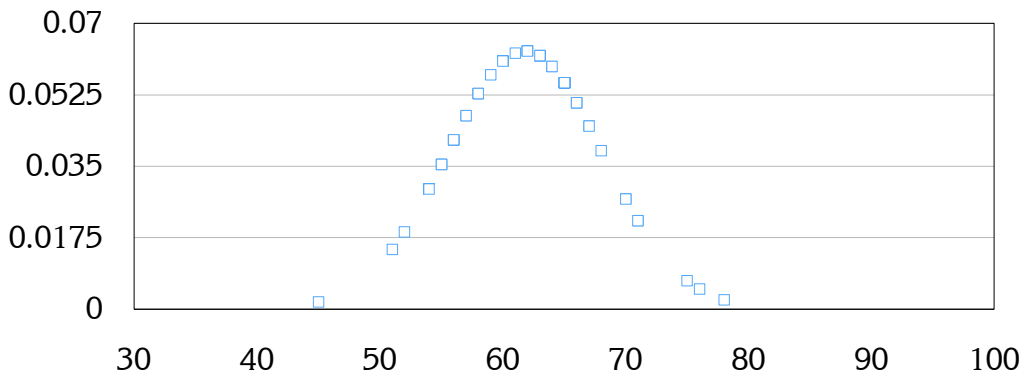

(Standard deviation 6.31mean 61.45) Standard Age Distribution In Our Patient

\begin{tabular}{|c|c|c|}
\hline Age group & Total & Percentage \\
\hline $41-45$ & 1 & 1.6 \\
\hline $46-50$ & 2 & 3.3 \\
\hline $51-55$ & 10 & 16.7 \\
\hline $56-60$ & 14 & 23.3 \\
\hline $61-65$ & 20 & 33.4 \\
\hline $66-70$ & 8 & 13.4 \\
\hline $71-75$ & 3 & 5.0 \\
\hline $76-80$ & 2 & 3.3 \\
\hline
\end{tabular}

1. From our study age distribution chart shows as age advances prevalence of osteoarthritis increases.

2. Mean age of our study group is $61.45 \mathrm{yrs}$, with minimum $45 \mathrm{yrs}$ and max $78 \mathrm{yrs}$.

3. Standard deviation of 6.31 .

4. Maximum incidence was in the age group 61-65 yrs.

5. Irrespective of age group females are affected more than males.

\section{Sex Distribution}

\section{SEX}

Male $\quad$ Female

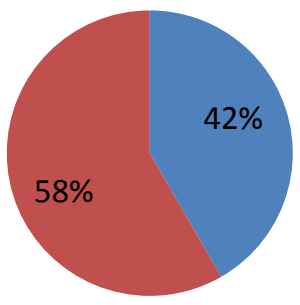

1. Out of 60 patients studied 25 are male and 35 are female.

2. There is more prevalence of osteoarthritis in female patients in our study.

\section{Bmi Distribution}

\begin{tabular}{|c|c|c|}
\hline Gender & Number & Percentage \\
\hline Male & 25 & 42 \\
\hline Female & 35 & 58 \\
\hline
\end{tabular}

In our study BMI ranges from 18.49 to 29.43 around $73.36 \%$ of our patient are the category of overweight and obesity.

Average BMI of males in our group is 23.97

Average BMI of females in our group is 24.40

In our study none of the patient had BMI more than 30 


\section{Standard Distribution Of Hscrp In Our Patient}

- Mean hsCRP level in our patient $4.0 \mathrm{mg} / \mathrm{dl}$

- hsCRP level in our patient ranges from $2.0 \mathrm{mg} / \mathrm{dl}$ to $7.0 \mathrm{mg} / \mathrm{dl}$

- hsCRP is more than that of healthy general population. $(>1.1 \mathrm{mg} / \mathrm{dl})$

Pain Distribution In Our Patient

\begin{tabular}{|c|c|}
\hline Pain Score & No. of Patient \\
\hline VAS 3 & 3 \\
\hline VAS 4 & 10 \\
\hline VAS 5 & 7 \\
\hline VAS 6 & 26 \\
\hline VAS 7 & 14 \\
\hline VAS 8 & 0 \\
\hline
\end{tabular}

- Most of our patients are symptomatic with pain range from mild, moderate and severe.

- Average pain score of our patient is 5.6 in VAS Scale

X-Ray - KI Grading Distribution

\begin{tabular}{|c|c|}
\hline X RAY GRADE KL & No. of patients \\
\hline GRADE 2 & 20 \\
\hline GRADE 3 & 26 \\
\hline GRADE 4 & 14 \\
\hline
\end{tabular}

- $\quad$ Many of our patients belong to grad 3KL Grading.

- $\quad$ Pain severity does not match with KL Grading.

- $\quad$ Deformity is present in Grade 3 and Grade 4 patient

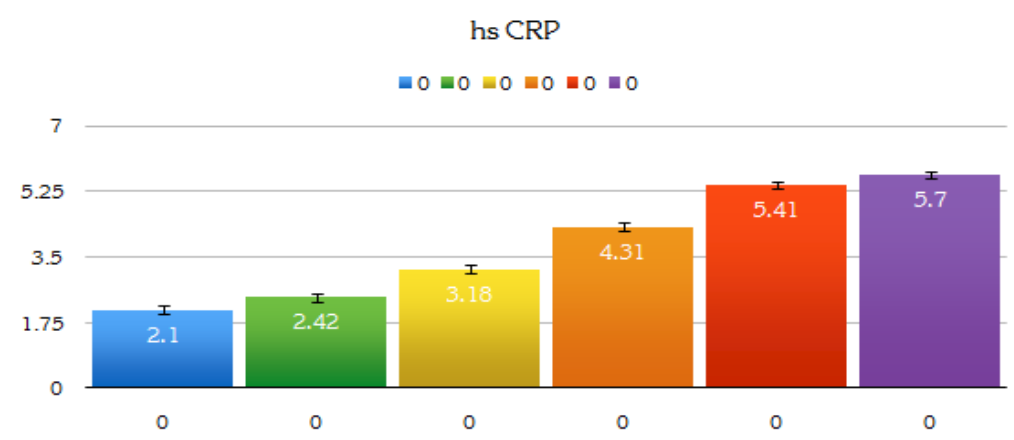

Scatter Graph Between Pain Vas And Hscrp

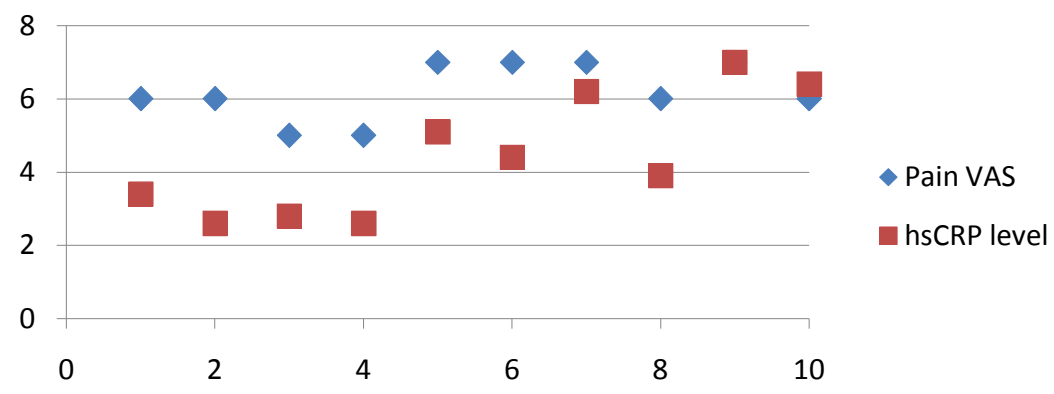

$\mathrm{X}=$ hsCRP

\begin{tabular}{|c|c|c|c|c|c|}
\hline \multirow{2}{*}{\multicolumn{6}{|c|}{$\begin{array}{l}\text { Standard weighted - means analysis } \\
\text { ANOVA Summary Independent Samples k=5 }\end{array}$}} \\
\hline & & & & & \\
\hline Source & SS & df & MS & $\mathbf{F}$ & $\mathbf{P}$ \\
\hline $\begin{array}{l}\text { Treatment } \\
\text { [between groups] }\end{array}$ & 71.8001 & 4 & 17.95 & 27.09 & $<.0001$ \\
\hline Error & 36.4433 & 55 & 0.6626 & & \\
\hline \multicolumn{6}{|l|}{$\mathrm{Ss} / \mathrm{BI}$} \\
\hline Total & 108.2434 & 59 & & & \\
\hline
\end{tabular}


Analysis Of Hscrp And Kl Grading

\begin{tabular}{|c|c|c|c|c|}
\hline Data Summary & \multicolumn{4}{|c|}{ Samples } \\
\hline & KL Grade 2 & KL Grade 3 & KL Grade 4 & Total \\
\cline { 2 - 5 } & 20 & 26 & 14 & 60 \\
\hline $\mathbf{N}$ & 73.5 & 115.9 & 51.6 & 241 \\
\hline $\mathbf{X}$ & 3.675 & 4.4577 & 3.6857 & 4.0167 \\
\hline Mean & 295.39 & 555.61 & 225.26 & 1076.26 \\
\hline$\square \mathbf{X}^{\mathbf{2}}$ & 1.3304 & 1.5585 & 2.6982 & 1.8346 \\
\hline Variance & 1.1534 & 1.2484 & 1.6426 & 1.3545 \\
\hline Std. Dev. & 0.2579 & 0.2448 & 0.439 & 0.1749 \\
\hline Std. Err. & & & & \\
\hline
\end{tabular}

\begin{tabular}{|c|c|c|c|c|c|}
\hline \multicolumn{6}{|c|}{ Standard weighted- means analysis } \\
\hline \multicolumn{6}{|c|}{ ANOVA SUMMARY Independent Samples k=3 } \\
\hline Source & SS & Df & MS & $\mathbf{F}$ & $\mathbf{P}$ \\
\hline $\begin{array}{l}\text { Treatment [between } \\
\text { groups] }\end{array}$ & 8.9252 & 2 & 4.4626 & 2.56 & 0.086166 \\
\hline Error & 99.3181 & 57 & 1.7424 & & \\
\hline \multicolumn{6}{|l|}{ Ss/BIs } \\
\hline Total & 108.2433 & 59 & & & \\
\hline
\end{tabular}

\section{Analysis Of Bmi And Hscrp}

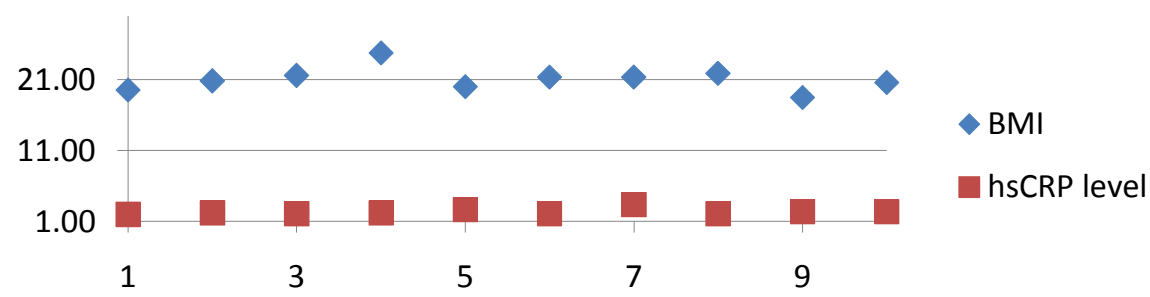

Pearsons Correlation Method

\begin{tabular}{|c|c|}
\hline $\begin{array}{c}\text { Result Details \& calculation } \\
\text { X values } \\
\Sigma=1453.19 \\
\text { Mean }=24.22 \\
\Sigma\left(\mathrm{X}-\mathrm{M}_{\mathrm{x}}\right)^{2}=\mathrm{SS}_{\mathrm{x}}=478.253 \\
\mathrm{Y} \text { values } \\
\Sigma=241 \\
\mathrm{Mean}^{2}=4.017 \\
\Sigma\left(\mathrm{Y}-\mathrm{M}_{\mathrm{y}}\right)^{2}=\mathrm{ss}_{\mathrm{y}}=108.243 \\
\mathrm{X} \text { and } \mathrm{Y} \text { combined } \\
\mathrm{N}=60 \\
\left.\Sigma\left(\mathrm{X}-\mathrm{M}_{\mathrm{x}}\right) \mathrm{Y}-\mathrm{M}_{\mathrm{y}}\right)=156.146 \\
\mathrm{R} \text { Calculation } \\
\mathrm{r}=\Sigma\left(\left(\mathrm{X}-\mathrm{M}_{\mathrm{y}}\right)\left(\mathrm{Y}-\mathrm{M}_{\mathrm{x}}\right)\right) / \\
\sqrt{\left(\left(\mathrm{SS}_{\mathrm{x}}\right)\left(\mathrm{SS}_{\mathrm{y}}\right)\right)} \\
\mathrm{r}=156.146 / \\
\sqrt{ }((478.253)(108.243))= \\
0.6863 \\
\mathrm{r}=0.6863\end{array}$ & $\begin{array}{l}\text { Key } \\
X: \text { BMI Values } \\
Y: \text { hsCRP Values } \\
M_{x}: \text { Mean of BMI Values } \\
M_{\mathrm{y}}: \text { Mean of hsCRP Values } \\
X-M_{\mathrm{x}} \& Y-\mathrm{M}_{\mathrm{y}}: \text { Deviation Scores } \\
\left(\mathrm{X}-\mathrm{M}_{\mathrm{x}}\right)^{2} \&\left(\mathrm{Y}-\mathrm{M}_{\mathrm{y}}\right)^{2}: \text { Deviation Squared } \\
\left(\mathrm{X}-\mathrm{M}_{\mathrm{x}}\right)\left(\mathrm{Y}-\mathrm{M}_{\mathrm{y}}\right): \text { Product of Deviation scores }\end{array}$ \\
\hline
\end{tabular}

The value of $\mathrm{R}$ is 0.6863 . This is a moderate positive correlation, which means there is a tendency for high $\mathrm{X}$ (BMI ) variable scores go with high Y (hsCRP) variable scores (and vice versa).

\section{Limitation of study}

- Our study size is small for comarision of for the large population.

- Extrems of BMI were not taken into account. 
- Our patients are symtamatic hence hsCRP levels in A-symptomatic patient or not included in our study.

- Our study did not have controles subject, hsCRP control level is taken general population.

\section{VII.Conclusion}

In our studyof hscrp levels in osteoarthritis patients the following conclusions are

- Osteoarthritis is more prevalent in females.

- hsCRP levels are modestly elevated in many patients particularly those with local symptoms .

- hsCRP levels rise with increasing pain scores.

- hsCRP levels do not correlate with X-ray grading .

- hsCRP levels have minimal correlation with body mass index.

- Patients with high levels of hscrp are likely to progress to advance disease.

\section{References}

[1]. Peyron JG: T epidemiology of osteoarthritis. In Moskowitz RW, Howell DS, Goldberg YM et al, editors: Osteoarthritis: diagnosis and management. Philadelphia, 1984, WB Saunders, pp 9-27.

[2]. Martin JA, Buckwalter JA: Aging, articular cartilage chondrocyte senescence and osteoarthritis, Biogerontolgy 3(5):257-264, 2001

[3]. Lawrence JS: Rheumatism in populations. London 1977, Heinemann Medical.

[4]. Anderson JJ, Felson DT: Factors associated with osteoarthritis of the knee in the first National Health and Nutrition Examination Survey (HANES I). Evidence for an association with overweight, race and physical demands of work, AM J Epidemiol 128(1):179$189,1988$.

[5]. Sokoloff L: The biology of degenerative joint disease. Chicago, 1969, University of Chicago Press.

[6]. Mankin HJ, Brandt KD: Biochemistry and metabolism of articular cartilage in osteoarthritis. In Moskowitz RW, Howell DS, Goldberg VM et al, editors: Osteoarthritis; diagnosis and medical/surgical management, ed 2, Philadelphia, 1992, WB Saunders, pp $109-154$.

[7]. Bruyere $\mathrm{O}$, Genant $\mathrm{H}$, Kothari $\mathrm{M}$ et al: Longitudinal stydy of magnetic resonance imaging and standard $\mathrm{Xi}$ rays to assess disease progression in osteoarthritis, Osteoarthritis Cartil 15(1): 98-103, 2007

[8]. Pelletier JP, Martel-Pelletier J, AbramsonSB: Osteoarthritis, an inflammatory disease: potential implication for the selection of new therapeutic targets, Arthritis Rheum 44(6): 1237-1247<2001.

[9]. Oldberg A, Antonsson P, Lindblom K, Heinegard D: COMP (cartilage Oligomeric matrix protein) is structurally related to the thrombospondins, J Biol Chem 267(31):22346-22350, 1992.

[10]. DiCesare PE, Morgelin M, Mann K, Paulsson M: Cartilage Oligomeric matrix protein and thrombospondin 1. Purification from articular cartilage, electron microscopic structure and chondrocyte binding, Eur J Biochem 223(3):927-937, 1994.

[11]. Hedbom E, Antonsson P, Hjerpe A et al: Cartilage matrix proteins. An acidic Oligomeric protein (COMP) detected only in cartilage, J Biol chem. 267(9):6132-6136, 1992.

[12]. Morgelin M, Heinegard D, Engel J, Paulsson M: Electron microscopy of native cartilage Oligomeric matrix protein purified from the swarm rat chondrosarcoma reveals a five-armed structutre, J Biol Chem 267(9):6137-6141, 1992.

[13]. Hedin PJ, Weitoft TY, Hedin H et al: Serum concentrations of hyaluronan and proteoglycan in joint disease. Lack of association, $\mathrm{J}$ Rheumatol 18(10):1601-1605, 1991.

[14]. Lawrence JS: Generalized osteoarthrosis in a population sample, Am J Epidemiol 90(5):381-389, 1969.

[15]. Burnett SJ, hart DJ, Cooper C, Spector TD: A radiographic atlas of osteoarthritis. London, 1994, Springer-Verlag.

[16]. Cooper C, Snow S, McAlindon TE et al: Risk factors for the incidence and progression of radiographic knee osteoarthritis, Arthritis Rheum 43:995-1000, 2000

[17]. Jessica W. Smith, Thomas B. Martins et al: Significance of C-reactive protein in osteoarthritisand total knee arthroplasty outcomes, Ther Adv Musculoskeletal Dis. 4(5): 315-325, 2012. 